TITLE:

Classification of projectively homogeneous surfaces(Algebraic Manipulation for Differential Equations)

$\operatorname{AUTHOR}(\mathrm{S}):$

SASAKI, TAKESHI

CITATION:

SASAKI, TAKESHI. Classification of projectively homogeneous surfaces(Algebraic Manipulation for Differential Equations). 数理解析研究所講究録 1990, 729: 32-49

ISSUE DATE:

1990-10

URL:

http://hdl.handle.net/2433/101941

RIGHT: 


\title{
Classification of projectively homogeneous surfaces
}

\author{
TAKESHI SASAKI \\ Department of Mathematics \\ Faculty of Integrated Arts and Sciences \\ Hiroshima University \\ （広息大・争念合科学）
}

\section{Introduction}

A surface in $\mathbf{P}^{3}$ is called projectively homogeneous (homogeneous in short) if a subgroup of $P G L_{4}$ acts transitively on this surface. Such surfaces are classified by $\mathrm{S}$. Lie relying on his group theory when the coefficient field is $\mathbb{C}[\mathbf{L}]$. In this note* we show how to classify such surfaces over the real field relying on the projective differential geometry of surfaces. A surface in $\mathbf{P}^{3}$ is locally seen as an immersion of a two-manifold with coordinates $(x, y)$ into the affine space with coordinates $z=\left(z^{1}, \cdots, z^{4}\right)$, which is transversal to rays through the origin. The coordinates $z^{i}$, as functions of $(x, y)$, satisfy a completely integrable system of linear differential equations of the form

$$
\left\{\begin{array}{l}
z_{x x}=\ell z_{x y}+a z_{x}+b z_{y}+p z \\
z_{y y}=m z_{x y}+c z_{x}+d z_{y}+q z .
\end{array}\right.
$$

Our method of classification is first to find projective scalar invariants of this system and then to integrate the corresponding systems using the fact that such invariants are automatically constant on homogeneous surfaces. Examination of homogeneity turns out to be easy and we get a table of such surfaces. A classification of unimodular-affinely homogeneous surfaces has been given by this method in [G].

In $\S 1$ we will review the projective differential geometric treatment of surfaces and relate it to the above system. In this respect, the Fubini-Pick invariant denoted by $F$ plays a fundamental role. Surfaces with $F \neq 0$ is classified in $\S 2$. If $F=0$, then surfaces are known to be ruled and its classification is given in $\S 3$. In $\S 4$ we give some remarks.

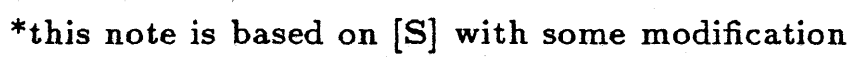




\section{$\S 1$. Projective differential geometry of surfaces.}

Let $M$ be a 2 -manifold immersed in a real projective space $\mathbf{P}^{3}$. Such an immersion has locally a lift to the affine space $A^{4}-\{0\}$. Let $e_{0}$ be one of lifts. Then we associate to each point of $M$ a set of four independent vectors $\left\{e_{0}, e_{1}, e_{2}, e_{3}\right\}$ with $e_{0}$ as the first vector so that this set moves smoothly on $M$. Such a set $e$, that is only locally defined, is called a projective frame field. Certainly we can assume $e$ is unimodular in the sense that $\operatorname{det}\left(e_{0}, e_{1}, e_{2}, e_{3}\right)=1$ with respect to a fixed volume form of $\mathrm{A}^{4}$. The dependence of $e$ on $M$ is denoted by a Pfaff system

$$
d e=\omega e \quad \text { i.e. } \quad d e_{\alpha}=\sum_{\beta} \omega_{\alpha}^{\beta} e_{\beta}
$$

which is our starting setting. The integrability condition is

$$
d \omega_{\alpha}^{\beta}=\sum_{\gamma} \omega_{\alpha}^{\gamma} \wedge \omega_{\gamma}^{\beta}
$$

Now we restrict freedom of choice of projective frame fields. First, we may assume $\omega_{0}^{3}=0$ so that the vector space $\left\langle e_{0}, e_{1}, e_{2}\right\rangle$ becomes the tangent space of the cone over $e_{0}(M)$ with the origin as the vertex. This choice implies

$$
0=d \omega_{0}^{3}=\omega_{0}^{\alpha} \wedge \omega_{\alpha}^{3}=\omega_{0}^{i} \wedge \omega_{i}^{3}
$$

Here we use the summation convention; repeated indices are summed up in their range and the range for $\alpha, \beta, \cdots$ is 0 to 3 and the range for $i, j, \cdots$ is 1 to 3 . We also use the notation $\omega^{i}$ for $\omega_{0}^{i}$. From (1.3) we see the existence of symmetric functions $h_{i j}$ so that

$$
\omega_{i}^{3}=h_{i j} \omega^{j}
$$

Put $\varphi_{2}=h_{i j} \omega^{i} \omega^{j}$. Then we can see the conformal class of $\varphi_{2}$ is independent of frames chosen (see Proposition 1.1). From now on we assume that this form is non-degenerate; an immersion or a surface with this assumption is called non-degenerate. We next take the exterior derivation of (1.4) to get the identity

$$
\left(d h_{i j}-h_{i k} \omega_{j}^{k}-h_{k j} \omega_{i}^{k}+h_{i j}\left(\omega_{0}^{0}+\omega_{3}^{3}\right)\right) \wedge \omega^{j}=0 .
$$




\section{4}

Hence we can put

$$
h_{i j k} \omega^{k}=d h_{i j}-h_{i k} \omega_{j}^{k}-h_{k j} \omega_{i}^{k}+h_{i j}\left(\omega_{0}^{0}+\omega_{3}^{3}\right)
$$

and we get a cubic form

$$
\varphi_{3}=h_{i j k} \omega^{i} \omega^{j} \omega^{k}
$$

DEFINITION. The scalar function

$$
F=h_{i j k} h_{p q r} h^{i p} h^{j q} h^{k r}, \quad\left(h^{i j}\right)=\left(h_{i j}\right)^{-1}
$$

is called the Fubini-Pick invariant.

We can see moreover that our consideration can be restricted to the case $\left|\operatorname{det}\left(h_{i j}\right)\right|=1$, which enables us to choose a frame with the condition

$$
\omega_{0}^{0}+\omega_{3}^{3}=0
$$

Then we see

Proposition 1.1. (1) Let $e$ and $\tilde{e}$ be two projective frame fields. Then they are related by a frame change

$$
\tilde{e}=g e \text { where } g=\left(\begin{array}{ccc}
\lambda & 0 & 0 \\
b & a & 0 \\
\mu & c & \nu
\end{array}\right),
$$

$a$ being a $2 \times 2$-matrix, with the property $|\operatorname{det} a|=|\lambda \nu|=1$.

(2) The forms $\varphi_{2}, \tilde{\varphi}_{2}, \varphi_{3}, \tilde{\varphi}_{3}$ and functions $F, \tilde{F}$ are related as

$$
\tilde{\varphi}_{2}=\lambda \nu^{-1} \varphi_{2}, \quad \tilde{\varphi}_{3}=\lambda \nu^{-1} \varphi_{3}, \quad \tilde{F}=\lambda^{-1} \nu F .
$$

Proposition 1.2. The coefficients $h_{i j k}$ satisfies the condition

$$
h^{i j} h_{i j k}=0 \text {. }
$$

This condition is called the apolarity condition.

We next take the exterior derivation of (1.5) to get the identity

$$
\left(\omega_{i}^{0}-h_{i j} \omega_{3}^{j}\right) \wedge \omega^{i}=0 .
$$

Hence we may put

$$
\omega_{i}^{0}-h_{i j} \omega_{3}^{j}=L_{i j} \omega^{j}
$$

Without proof we cite a 
Proposition 1.3. Assume the immersion is non-degenerate. Then there exists locally a projective frame field with the property

$$
\omega_{0}^{3}=\omega_{0}^{0}+\omega_{3}^{3}=0, \quad\left|\operatorname{det}\left(h_{i j}\right)\right|=1, \quad h^{i j} L_{i j}=0 .
$$

Such frames are related by a transformation $e \rightarrow \tilde{e}=g e$ where components of $g$, denoted as in Proposition 1.1, satisfy $|\operatorname{det} a|=|\lambda \nu|=1, b=\nu^{-1} a h^{t} c$, and $\mu=\frac{1}{2} \nu^{-1} c h^{t} c$.

We finally introduce the last invariant $\gamma_{i}$ by

$$
\omega_{3}^{0}=-\gamma_{i} \omega^{i} .
$$

The covariance of the invariants are seen to be as follows:

$$
\begin{gathered}
\lambda^{2} \nu \tilde{h}_{i j k}=h_{p q r} a_{i}^{p} a_{j}^{q} a_{k}^{r} \\
\lambda^{2} \tilde{L}_{i j}=\left(L_{k \ell}-\lambda h_{k \ell m} c^{m}\right) a_{i}^{k} a_{j}^{\ell} \\
\lambda^{2} \tilde{\gamma}_{j}=\left(\nu \gamma_{k}+c^{i} L_{i k}-\frac{1}{2} \nu^{-1} c^{i} c^{\ell} h_{i \ell k}\right) a_{j}^{k}
\end{gathered}
$$

For later use we cite a

TheOREM 1.4. A non-degenerate surface with $\varphi_{3}=0$ is a quadratic surface. A non-degenerate surface is ruled if and only if the invariant $F$ vanishes.

For a precise treatment of the above materials with proofs, refer the notes [S].

\section{$\S 2 . H o m o g e n e o u s$ surfaces with $F \neq 0$.}

2.1 Let us consider homogeneous surfaces whose Fubini-Pick invariant $F$ does not vanish. To such a surface we associate a distinguished frame that will now be constructed. Take a normalized frame with

$$
\omega_{0}^{0}+\omega_{3}^{3}=0 \text { and } h^{i j} L_{i j}=0
$$


and satisfying $h=\left(\begin{array}{cc}1 & 0 \\ 0 & \varepsilon\end{array}\right), \varepsilon= \pm 1 ;$ i.e.

$$
\omega_{1}^{3}=\omega^{1}, \quad \omega_{2}^{3}=\varepsilon \omega^{2} .
$$

The apolarity condition is $h_{111}+\varepsilon h_{122}=h_{112}+\varepsilon h_{222}=0$. By the formula (1.6), we find a frame with

$$
h_{111}=-\varepsilon h_{122}=-2 \text { and } h_{112}=h_{222}=0
$$

Then, by definition of the cubic form,

$$
\omega_{1}^{1}=\omega^{1}, \quad \omega_{2}^{2}=-\omega^{1} \quad \text { and } \quad \omega_{2}^{1}+\varepsilon \omega_{1}^{2}=-2 \varepsilon \omega^{2}
$$

Note here that $\tilde{\omega}_{0}^{0}=\omega_{0}^{0}-b_{i} \omega^{i}$ by a frame change when $\lambda=1$ and $a=I_{2}$ in the notation of $\S 1$. Hence we can assume

$$
\omega_{0}^{0}=\omega_{3}^{3}=0
$$

Now it is important to see that there exist only finite frames satisfying (2.1) to (2.5). Therefore, if the surface is homogeneous and $F \neq 0$, then the coefficients of remaining components of $\omega$ must be constant. We define coefficients as follows:

$$
\begin{array}{ll}
\omega_{1}^{2}=a \omega^{1}+(b-1) \omega^{2}, & \varepsilon \omega_{2}^{1}=-a \omega^{1}-(b+1) \omega^{2}, \\
\omega_{1}^{0}=p \omega^{1}+q \omega^{2}, & \omega_{2}^{0}=q \omega^{1}+r \omega^{2}, \\
\omega_{3}^{1}=p^{\prime} \omega^{1}+q^{\prime} \omega^{2}, & \omega_{3}^{2}=\varepsilon q^{\prime} \omega^{1}+\varepsilon r^{\prime} \omega^{2}, \\
\omega_{3}^{0}=u \omega^{1}+v \omega^{2} . &
\end{array}
$$

Here we have used the fact that, if we put $\omega_{i}^{0}=p_{i j} \omega^{j}$ and $h_{i j} \omega_{3}^{j}=q_{i j} \omega^{j}$, then $\left(p_{i j}\right)$ and $\left(q_{i j}\right)$ are symmetric matrices in view of (2.5). Assume now the coefficients are constant. The integrability condition $d \omega=\omega \wedge \omega$ is 
written in terms of these coefficients as follows:

$$
d \omega^{1}=\varepsilon a \omega^{1} \wedge \omega^{2}, \quad d \omega^{2}=b \omega^{1} \wedge \omega^{2}
$$

$$
p^{\prime}+\varepsilon r^{\prime}=p+\varepsilon r, \quad q^{\prime}-q=3 \varepsilon a
$$

$$
\begin{gathered}
p+\varepsilon r^{\prime}=\varepsilon a^{2}+(b-1)(b-2), p^{\prime}+\varepsilon r=\varepsilon a^{2}+(b+1)(b+2) \\
\varepsilon a(p-\varepsilon r)+2(b-1) q=v, \varepsilon a\left(p^{\prime}-\varepsilon r^{\prime}\right)+2(b+1) q^{\prime}=-v \\
(b-1)\left(p^{\prime}-\varepsilon r^{\prime}\right)-2 a q^{\prime}=-u, \quad(b+1)(p-\varepsilon r)-2 a q=u \\
q\left(p^{\prime}-\varepsilon r^{\prime}\right)-q^{\prime}(p-\varepsilon r)=\varepsilon a u+b v
\end{gathered}
$$

In order to solve this system (2.8) put $2 t=p^{\prime}+\varepsilon r^{\prime}=p+\varepsilon r, 2 k=p-\varepsilon r$ and $p^{\prime}-\varepsilon r^{\prime}=2 k^{\prime}$. Then from (2.8.2), $2 t=\varepsilon a^{2}$ and $k-k^{\prime}=-3 b$. From (2.8.3), $\varepsilon a\left(k+k^{\prime}+3\right)+b\left(q+q^{\prime}\right)=0$ and from (2.8.4), $a\left(q+q^{\prime}\right)-b\left(k+k^{\prime}-3\right)=$ 0 . Hence $6 a b+\left(a^{2}+\varepsilon b^{2}\right)\left(q+q^{\prime}\right)=0$. Assume here $a \neq 0$ and $b \neq 0$. Then $a^{2}+\varepsilon b^{2} \neq 0$. Combining with (2.8.1), we have $2 q=-3 \varepsilon a-6 a b /\left(a^{2}+\varepsilon b^{2}\right)$, $2 q^{\prime}=3 \varepsilon a-6 a b /\left(a^{2}+\varepsilon b^{2}\right), 2 k=-3 b+3\left(\varepsilon b^{2}-a^{2}\right) /\left(a^{2}+\varepsilon b^{2}\right), 2 k^{\prime}=$ $3 b+3\left(\varepsilon b^{2}-a^{2}\right) /\left(a^{2}+\varepsilon b^{2}\right):$ and consequently, $v=-6 a b\left(\varepsilon a^{2}+b^{2}-\right.$ 1) $/\left(a^{2}+\varepsilon b^{2}\right)$ and $u=3\left(a^{2}-\varepsilon b^{2}\right)\left(\varepsilon a^{2}+b^{2}-1\right) /\left(a^{2}+\varepsilon b^{2}\right)$. Now (2.8.5) shows $\left(3 b^{2}-\varepsilon a^{2}\right)\left(\varepsilon a^{2}+b^{2}-4\right)=0$. So we have two cases $3 b^{2}=a^{2}$ or $\varepsilon a^{2}=4-b^{2}$. However the first case is reduced to the case where $a=0$. This is seen by the transformation rule (1.6) taking $\lambda=1$ and $a=\left(\begin{array}{cc}\cos \theta & \sin \theta \\ -\sin \theta & \cos \theta\end{array}\right)$ for $\theta=2 \pi / 3$. Note that this transformation keeps the condition (2.3). Therefore it is enough to consider $\varepsilon a^{2}+b^{2}=4$ when $a \neq 0$ and $b \neq 0$. When one of $a$ and $b$ is zero, the computation is easier and thus we have the following list of solutions: 


\begin{tabular}{|c|c|c|c|}
\hline & {$[1]$} & {$[2]$} & {$[3]$} \\
\hline$a$ & $a \neq 0$ & 0 & 0 \\
\hline$b$ & $a^{2}+\varepsilon b^{2}=4 \varepsilon$ & $b \neq 0$ & 0 \\
\hline$p$ & $\frac{1}{2}-\frac{3}{2} b+\frac{1}{4} b^{2}$ & $\frac{3}{2}(1-b)$ & $p$ \\
\hline$q$ & $-\frac{\jmath}{2} \varepsilon a-\frac{\jmath}{4} \varepsilon a b$ & 0 & $q$ \\
\hline$\varepsilon r$ & $\frac{7}{2}+\frac{3}{2} b-\frac{5}{4} b^{2}$ & $-\frac{3}{2}(1-b)$ & - \\
\hline$p^{\prime}$ & $\frac{1}{2}+\frac{3}{2} b+\frac{1}{4} b^{2}$ & $\frac{3}{2}(1+b)$ & $p$ \\
\hline$q^{\prime}$ & $\frac{3}{2} \varepsilon a-\frac{3}{4} \varepsilon a b$. & 0 & $q$ \\
\hline$\varepsilon r^{\prime}$ & $\frac{7}{2}-\frac{3}{2} b-\frac{5}{4} b^{2}$ & $-\frac{3}{2}(1+b)$ & - \\
\hline$u$ & $9\left(1-\frac{1}{2} b^{2}\right)$ & $3\left(1-b^{2}\right)$ & $2 p$ \\
\hline$v$ & $-\frac{9}{2} \varepsilon a b$ & 0 & $-2 q$ \\
\hline
\end{tabular}

2.2 The surface corresponding to each solution is obtained by solving a Pfaffian equation $d e=\omega e$. We first carry out the integration for the case [2]. Since $d \omega^{1}=0$ and $d \omega^{2}=b \omega^{1} \wedge \omega^{2}$, there is a local coordinate system $(x, y)$ so that $\omega^{1}=d x$ and $\omega^{2}=e^{b x} d y$. Let $z=e_{0}$. Then $d e_{0}=\omega^{1} e_{1}+\omega^{2} e_{2}$ shows $e_{1}=z_{x}$ and $e_{2}=z_{y} e^{-b x}$. Since $d e_{1}=z_{x x} d x+z_{x y} d y=\omega_{1}^{\alpha} e_{\alpha}$ and 
$d e_{2}=\left(e^{-b x} z_{y}\right)_{x} d x+\left(e^{-b x} z_{y}\right)_{y} d y=\omega_{2}^{\alpha} e_{\alpha}$, we have

$$
\begin{aligned}
& z_{x x}=\frac{3}{2}(1-b) z+z_{x}+e_{3} \\
& z_{x y}=(b-1) z_{y} \\
& z_{y y} e^{-2 b x}=\frac{3}{2} \varepsilon(b-1) z-\varepsilon(b+1) z_{x}+\varepsilon e_{3} .
\end{aligned}
$$

Hence, putting $z=e^{(b-1) x} w$ and cancelling $e$, we have

$$
\begin{aligned}
& w_{x y}=0 \\
& w_{x x}-\varepsilon e^{-2 b x} w_{y y}=(4-b) w_{x} .
\end{aligned}
$$

It is easy to integrate this system and to obtain solutions:

$$
\begin{aligned}
& \left\{1, y, e^{(4-b) x}, \quad \frac{y^{2}}{2}+\frac{\varepsilon}{2 b(b+4)} e^{-2 b x}\right\}, \\
& \left\{1, y, x, \quad \frac{y^{2}}{2}+\frac{\varepsilon}{64} e^{-8 x}\right\}, \\
& \left\{1, y, e^{8 x}, \quad \frac{y^{2}}{2}+\frac{\varepsilon}{8} x e^{8 x}\right\},
\end{aligned}
$$

when $|b| \neq 4, b=4$ and $b=-4$ respectively. Then the corresponding surfaces are respectively

$$
\begin{array}{rlrl}
1^{o} & Z & =Y^{2}+\varepsilon X^{k} \quad k \neq 0,1,2 \\
2^{\circ} & Z & =Y^{2}+\varepsilon e^{X} \\
3^{\circ} & Z & =Y^{2}+\varepsilon X \log X
\end{array}
$$

$(X, Y, Z)$ being certain affine coordinates. These are all homogeneous.

2.3 The case [3] is similarly treated. Since $d \omega^{1}=d \omega^{2}=0$, we may put $\omega^{1}=d x$ and $\omega^{2}=d y$. The system for $z=e_{0}$ is $z_{x x}-\varepsilon z_{y y}=2 p z+2 z_{x}$ and $z_{x y}=q z-z_{y}$. Introducing a new variable $w$ by $z=e^{-x} w$ and writing $(x, y)$ for $\left(\frac{1}{2} x, \frac{1}{2} y\right)$, we have

$$
\left\{\begin{aligned}
w_{x x}-\varepsilon w_{y y} & =p w+2 w_{x} \\
w_{x y} & =q w
\end{aligned}\right.
$$


where $p$ and $q$ are new constants. This system is equivalent to the system considered by Wilczynski in $[\mathbf{W}]$ when $\varepsilon=-1$. We follow his argument with a little modification.

First assume $q=0$. Then the surface is a translation surface $w=f(x)+$ $g(y)$, where $f$ and $g$ are determined by $f_{x x}-2 f_{x}-p f=\varepsilon g_{y y}+p q=$ const. Since the characteristic equations are $(\rho-1)^{2}=p+1$ and $\rho^{2}=-\varepsilon p$, we must consider five cases separately: $p<-1, p=-1,-1<p<0$, $p=0$ and $p>0$. Let $p<-1$ and $\varepsilon=-1$. Then the solutions are $\left\{e^{x} \cos \lambda x, e^{x} \sin \lambda x, \cos \mu y, \sin \mu y\right\}$ where $\lambda^{2}=-p-1$ and $\mu^{2}=-p$. This yields the surface $\left(Y^{2}+Z^{2}\right) /\left(1+X^{2}\right)=\exp \left(k \tan ^{-1} X\right)$ for $X=$ $\tan \lambda x, Y=e^{-x} \cos \mu y / \cos \lambda x$ and $Z=e^{-x} \sin \mu y / \cos \lambda x . \quad k=-2 / \lambda$. When $\varepsilon=1$, solutions are $\left\{e^{x} \cos \lambda x, e^{x} \sin \lambda x, e^{\mu y}, e^{-\mu y}\right\}$, which yields $\left(Y^{2}+Z^{2}\right) / X=\exp \left(k \tan ^{-1} Z / Y\right)$ where $k=2 / \lambda$. This is equivalent to $Y Z /\left(1+X^{2}\right)=\exp \left(k \tan ^{-1} X\right)$ by a projective transformation. So both are together written as

$$
4^{\circ} \quad \tan ^{-1} Z=k \log \left(\frac{X^{2}-\varepsilon Y^{2}}{1+Z^{2}}\right) \quad k \neq 0
$$

by a trivial change of coordinates. Remaining four cases are similarly integrated and we have

$$
\begin{aligned}
& 5^{\circ} \quad e^{Z}=X^{2}+\varepsilon Y^{2} \quad(p=-1) \\
& 6^{\circ} \quad Z^{k}=X^{2}-Y^{2} \quad k>2 \text { when } \varepsilon=1 \text { and } 2>k>1 \text { when } \varepsilon= \\
& -1 \quad(-1<p<0) \\
& 7^{\circ} \quad Z=Y^{2}-\varepsilon \log X \quad(p=0) \\
& 8^{\circ} \quad Z^{k}=X^{2}+Y^{2} \quad k>2 \text { when } \varepsilon=-1 \text { and } 2>k>1 \text { when } \varepsilon= \\
& 1 \quad(p>0) .
\end{aligned}
$$

These are all homogeneous; for example, the surface $4^{\circ}$ has two sets of rotations when $\varepsilon=-1$ and spiral motions in $x$ and translations in $y$ when $\varepsilon=1$.

We next consider the case $q \neq 0$. The system has $e^{a x+b y}$ as a solution where $a^{2}-\varepsilon b^{2}=p+2 a$ and $a b=q$; hence

$$
a^{4}-2 a^{3}-p a^{2}-\varepsilon q^{2}=0
$$


Types of solutions of this algebraic equations are divided into six cases: (i) $(r, r, r, s)$, (ii) $(r, r, \alpha, \bar{\alpha})$, (iii) $(r, r, s, t)$, (iv) $(\alpha, \bar{\alpha}, \beta, \bar{\beta}),(\mathrm{v})(r, s, \alpha, \bar{\alpha})$ and (vi) $(r, s, t, u)$ where $r, s, t, u$ are different reals and $\alpha, \beta$ are non-real different complex numbers. Other combinations do not occur because of $q \neq 0$. The case (i) occurfs only when $p=-9 / 8, q=3 \sqrt{3} / 16$ and $\varepsilon=1$. The solutions are $\left\{1,3 x-\sqrt{3} y, 8 \sqrt{3} y+(3 x-\sqrt{3} y)^{2}, e^{-x-\sqrt{y}}\right\}$. However this yields a surface equivalent to $7^{\circ}$; so excluded. The solutions of (ii) are $\left\{e^{r x+b y},(r x-b y) e^{r x+b y}, e^{-\lambda^{2} x-b y} \cos \left(\lambda x-\frac{b}{\lambda} y\right), e^{-\lambda^{2} x-b y} \sin \left(\lambda x-\frac{b}{\lambda} y\right)\right\}$ where $b=q / r$ and $\lambda^{2}=a-1>0$. This represents

$$
9^{\circ} \quad Z+\log \left(X^{2}+Y^{2}\right)=k \tan ^{-1} \frac{Y}{X}, \quad k>0 \quad(\varepsilon=-1) .
$$

For the case (iii) it is seen that we may put $r=1-\lambda^{2}, s=\lambda-\lambda^{2}$ and $t=-\lambda-\lambda^{2}$, when $|\lambda| \neq 1$. Since solutions are $\left\{e^{r x+b y},(r x-b y)\right.$ $\left.e^{r x+b y}, e^{s x+\frac{q}{s} y}, e^{t x+\frac{q}{t} y}\right\}(b=r / q)$, the surface is

$10^{\circ} \quad Z=\frac{1}{\lambda-1} \log X-\frac{1}{\lambda+1} \log Y,\left(\varepsilon=1\right.$ when $\lambda^{2}>1 ; \varepsilon=-1$ when $\left.\lambda^{2}<1\right)$.

In the case (iv) the independent solutions over $\mathbb{C}$ are

$$
\left\{\exp \left((a \pm i \lambda) x+\frac{q}{a \pm i \lambda} y\right), \exp \left((1-a \pm i \mu) x+\frac{1}{1-a \pm i \mu} y\right)\right\}
$$

for some parameters $\lambda$ and $\mu$ ( $a$ is a function of $\lambda$ and $\mu$ ). Taking real solutions we see

$$
11^{\circ} \quad \log \frac{X^{2}+Y^{2}}{1+Z^{2}}=k \tan ^{-1} \frac{Y}{X}+\ell \tan ^{-1} Z,
$$

$k$ and $\ell$ being real parameters determined by $\lambda$ and $\mu$. In the case (v) solutions are $\left\{\exp \left((a \pm i \lambda) x+\frac{q}{a \pm i \lambda} y\right), \exp \left(r x+\frac{q}{r} y\right), \exp \left(s x+{ }_{s}^{q} y\right)\right\} ; r$ and $s$ are functions of $a$ and $\lambda$. The corresponding surface is

$12^{\circ} \quad \log Z=k \tan ^{-1} \frac{Y}{X}+\frac{\ell}{2} \log \left(X^{2}+Y^{2}\right)$.

The last case (vi) yields

$13^{\circ}$

$$
Z=X^{k} Y^{\ell}
$$

We here do not try to determine exact ranges of $k$ and $\ell$ in $11^{\circ}, 12^{\circ}$ and 
$13^{\circ}$. For non-degeneracy it is necessary to assume $k \ell \neq 0$ and $\ell \neq 1$ for $12^{\circ}$ and $k \ell(k+\ell-1) \neq 0$ for $13^{\circ}$. Above surfaces are also homogeneous.

2.4 We integrate the final case [1]. Choose coordinates $(x, y)$ such that $\omega^{1}=$ $e^{a b x} d y$ and $\varepsilon \omega^{2}=\frac{b}{a} e^{a b x} d y-b d x$. Put $z=e_{0}$. Then $e_{1}=e^{-a b x} z_{y}+\frac{1}{a} z_{x}$ and $e_{2}=-\frac{\varepsilon}{b} z_{x}$. Hence we have

$$
\left\{\begin{array}{l}
u z_{x y}+\frac{1}{a} z_{x x}=(b-1) z_{x}+a b u z_{y}+\frac{3}{4} a b(b+2) z \\
u^{2} z_{y y}+\frac{4}{a b^{2}} u z_{x y}=\frac{4}{a b^{2}}\left(b^{2}-1\right) z_{x}+\frac{2}{b}(b+2) u z_{y}+\frac{6}{b}\left(1-b^{2}\right) z
\end{array}\right.
$$

where $u=e^{-a b x}$. Referring that $e^{\frac{3}{2} a b x}$ is a solution, put $z=e^{\frac{3}{2} a b x} w$. Then, for $v=y$, we see

$$
\left\{\begin{aligned}
u\left(w_{u u}-w_{v v}\right) & =(b+1) w_{u}-w_{v} \\
b u\left(w_{u u}-2 w_{v v}\right) & =(b+1) w_{u}-w_{v}
\end{aligned}\right.
$$

The independent solutions are

$$
\begin{aligned}
& w_{0}=1, \quad w_{1}=u+(b+1) v, \quad w_{2}=u^{2}-2 u v-(b+1) v^{2} \\
& w_{3}=u^{3}+(b-1) u^{2} v-(b-1) u v^{2}+\left(1-b^{2}\right) v^{3} / 3
\end{aligned}
$$

The corresponding equation of the surface is

$$
\left((b+1) w_{2}+w_{1}^{2}\right)^{3}=\frac{9}{16}(b+2)\left((b+1)^{2} w_{3}-\frac{2}{3}(b-1) w_{1}^{3}-\left(b^{2}-1\right) w_{1} w_{2}\right)^{2}
$$

when $|b| \neq 1,2$ and called Enriques surface. By a projective change of coordinates this is written

$$
14^{\circ} \quad\left(Y-X^{2}\right)^{3}=k\left(Z-\frac{2}{3} X^{3}-X Y\right)^{2}, k \neq 0, \frac{9}{4} .
$$

The case $|b|=1$ is included in $1^{\circ}$. The case $|b|=2$ is excluded because $a \neq 0$. However the case $b=2$ is interesting because it yields a surface of degree 4: $27 Z^{2}-X^{2} Y^{2}-42 X^{4}-4 Y^{3}-18 X Y Z=0$, which is known to be a homogeneous developable surface. 


\section{§3. Homogeneous surfaces with $F=0$.}

3.1 This section treats the case $F=0$. Since the surface is quadratic when the cubic form vanishes (THEOREM 1.4), we here consider surfaces with $F=0$ but with non-vanishing cubic form. Such a surface is ruled. Since it must be of indefinite type, we start with a normalized frame with $h=\left(\begin{array}{c}1 \\ 1\end{array}\right)$. We first see the existence of a frame with the following property:

$$
\begin{aligned}
& \omega_{1}^{3}=\omega^{2}, \quad \omega_{2}^{3}=\omega^{1}, \quad \omega_{1}^{2}=\omega^{1}, \quad \omega_{2}^{1}=0 \\
& h_{111}=-2, \quad h_{112}=h_{122}=h_{222}=0 \\
& \omega_{0}^{0}-3 \omega_{1}^{1}=0, \quad L_{i j}=0 \quad \text { and } \quad \gamma_{2}=0 .
\end{aligned}
$$

In fact, by the identity $F=2 h_{111} h_{222}$ we may assume $h_{222}=0$. The identity $h_{112}=h_{122}=0$ follows from the form of $h$. Consider a frame change by a transformation $g$ whose components are $\lambda \nu=1, a=\left(\begin{array}{cc}\alpha & 0 \\ 0 & \alpha^{-1}\end{array}\right)$, $b_{1}=\lambda c^{2}, b_{2}=\lambda c^{1}$ and $\mu=\lambda c^{1} c^{2}$. Then $h_{111}$ transforms as $\lambda \tilde{h}_{111}=h_{111} \alpha^{3}$. So there exist $\lambda$ and $\alpha$ so that $h_{111}=-2$; and this condition restricts frame changes to the case $\lambda=\alpha^{3}$. Then computation shows $\tilde{\omega}_{0}^{0}-3 \tilde{\omega}_{1}^{1}=$ $\omega_{0}^{0}-3 \omega_{1}^{1}+\lambda\left(-4 c^{2} \omega^{1}+2 c^{1} \omega^{2}\right)$. Thus we can find a frame with $\omega_{0}^{0}-3 \omega_{1}^{1}=0$. The identities $\omega_{1}^{2}=\omega^{1}$ and $\omega_{2}^{1}=0$ follow from the form of the cubic form. Taking exterior derivation of $\omega_{2}^{1}=0$, we see $0=L_{22} \omega^{1} \wedge \omega^{2}$, which shows $L_{22}=0$. Similarly $L_{11}=0$ is seen by derivation of $\omega_{1}^{2}=\omega^{1}$. Also the exterior derivation of $\omega_{2}^{0}-\omega_{3}^{1}=0$ shows $\gamma_{2}=0$. Notice here that frame changes which keep the condition (3.1) are now restricted to

$$
g(\alpha)=\left(\begin{array}{cccc}
\alpha^{3} & & & \\
& \alpha & & \\
& & \alpha^{-1} & \\
& & & \alpha^{-3}
\end{array}\right)
$$

We put

$$
\begin{aligned}
& \omega_{1}^{1}=a \omega^{1}+b \omega^{2}, \quad \omega_{1}^{0}=\omega_{3}^{2}=p \omega^{1}+q \omega^{2} \\
& \omega_{2}^{0}=\omega_{3}^{1}=r \omega^{1}+s \omega^{2}, \quad \omega_{3}^{0}=t \omega^{1}
\end{aligned}
$$

Coefficients change under $g(\alpha)$ as follows

$$
\tilde{p}=\alpha^{-4} p, \quad \tilde{q}=\alpha^{-6} q, \quad \tilde{r}=\alpha^{-6} r, \quad \tilde{s}=\alpha^{-8} s, \quad \tilde{t}=\alpha^{-8} t
$$


The integrability condition $d \omega=\omega \wedge \omega$ induces the following identities

$$
\begin{gathered}
d \omega^{1}=-2 b \omega^{1} \wedge \omega^{2}, \quad d \omega^{2}=4 a \omega^{1} \wedge \omega^{2}, \\
r=-2 q, q-r=3\left(b_{1}-a_{2}\right)+6 a b, \quad 4 b p-6 a q=q_{1}-p_{2}, \\
6 r b-8 a s=s_{1}-r_{2}, \quad s=t, \quad 6 b t=-t_{2}
\end{gathered}
$$

where indices imply the derivation with respect to $\omega^{1}$ and $\omega^{2}: d a=a_{1} \omega^{1}+$ $a_{2} \omega^{2}$.

3.2 Now assume $t \neq 0$. Then by (3.4) we may assume further $|t|=1$. So, if the surface is homogeneous, remaining coefficients are assumed to be constant. Then from (3.6), $a=b=q=r=0$. Next assume $s=t=0$. If $r \neq 0$, then by a similar reasoning $a, b, p$ and $q$ are constant. But then $b=0$ and following $r=0$ by (3.6), which is a contradiction. Thus $r=0$. When $b \neq 0$, we have $|b|=1$ and $a=p=0$. When $b=0$, we have either $a=0$ and $p=0,1,-1$ or $|a|=1$ and $p$ is arbitrary. Therefore homogeneous ruled surfaces are among four cases:

[4] $|t|=1, \quad a=b=q=r=0$

[5] $\quad p=0$ or $\pm 1, \quad a=b=q=r=s=t=0$

[6] $|a|=1, \quad b=q=r=s=t=0$.

[7] $|b|=1, \quad a=p=b=q=r=s=t=0$.

We first examine the case [7]. In this case $d \omega^{2}=0$ by (3.5) and consequently all diagonal forms are closed. Hence there is a frame change $g(\alpha)$ which transforms the coframe into

$$
\left(\begin{array}{cccc}
0 & \omega^{1} & \omega^{2} & 0 \\
& 0 & \omega^{1} & \omega^{2} \\
& & 0 & \omega^{1} \\
0 & & & 0
\end{array}\right)
$$

Moreover the same reasoning applies to the case [6] when $p=0$. Thus the case [7] is a subcase of [6]. 
3.3 We next integrate the case [5]. Since $\omega^{1}=d x$ and $\omega^{2}=d y$ for some local coordinates $(x, y)$, the associate system of differential equations is

$$
\left\{\begin{array}{l}
z_{x x}=p z+z_{y} \quad p=0, \pm 1 \\
z_{y y}=0 .
\end{array}\right.
$$

When $p=1$, the independent solutions are $\left\{e^{-x}, e^{x},(x+2 y) e^{x},(x-\right.$ $\left.2 y) e^{-x}\right\}$ and the surface is

$15^{\circ}$

$$
Z=X Y+X \log X
$$

When $p=-1$, the independent solutions are $\{\cos x, \sin x, x \sin x+$ $2 y \cos x, x \cos x-2 y \sin x\}$ and the surface is

$16^{\circ}$

$$
Z=X Y+\left(1+X^{2}\right) \tan ^{-1} X .
$$

When $p=0$, we get the Cayley surface

$17^{\circ}$

$$
Z=X Y+\frac{1}{3} X^{3}
$$

3.4 For the case [6] we choose coordinates $(x, y)$ such that $\omega^{1}=d x$ and $\omega^{2}=e^{4 a x} d y$; see (3.5). The system of differential equations for $z=e_{0}$ is

$$
\left\{\begin{array}{l}
z_{x x}=(p-3) z+4 a z_{x}+e^{-4 a x} z_{y} \\
z_{y y}=0
\end{array}\right.
$$

Putting $z=e^{2 a x} w$, we have $w_{x x}=(p+1) w+e^{-4 a x} w_{y}$ and $w_{y y}=0$. Hence $w=u(x)+y v(x)$. By a simple change of variables we see

$$
\left\{\begin{array}{l}
u_{x x}=p u+e^{x} v \\
v_{x x}=p v
\end{array}\right.
$$

$p$ is a new constant. We have four subcases according as $p>0$ and $p \neq 1 / 4$, $p=1 / 4, p<0$, or $p=0$. The space of independent solutions is respectively in this order $\left\{e^{-\lambda x}, e^{\lambda x}, \frac{1}{2 \lambda+1} e^{(\lambda+1) x}+y e^{\lambda x}, \frac{1}{1-2 \lambda} e^{(1-\lambda) x}+y e^{-\lambda x}\right\}$ where $p=\lambda^{2} ;\left\{e^{-\frac{1}{2} x}, e^{\frac{1}{2} x}, \frac{1}{2} e^{\frac{3}{2} x}+y e^{\frac{1}{2} x}, x e^{\frac{1}{2} x}+y e^{-\frac{1}{2} x}\right\} ;\left\{1, x, e^{x}+y,(x-2) e^{x}+\right.$ $x y\}$ and $\left\{\cos \lambda x, \sin \lambda x, a e^{x}(\cos \lambda x+2 \lambda \sin \lambda x)+y \cos \lambda x, a e^{x}(\sin \lambda x-\right.$ $2 \lambda \cos \lambda x)+y \sin \lambda x\}$ where $p=-\lambda^{2}$ and $a=\left(1+4 \lambda^{2}\right)^{-1}$. Therefore the corresponding surfaces are respectively. 


$$
\begin{array}{ll}
18^{\circ} & Z=X Y+X^{k}, \quad k \neq 0,2 \\
19^{\circ} & Z=X Y+X^{2} \log X \\
20^{\circ} & Z=X Y+e^{X} \\
21^{\circ} & \log \frac{X+Y Z}{1+Z^{2}}=k \tan ^{-1} Z, \quad k>0 .
\end{array}
$$

3.5 For the final case [4], the system is easily seen to be

$$
\left\{\begin{array}{l}
z_{x x}=p z+z_{y} \\
z_{y y}=t z \quad t= \pm 1
\end{array}\right.
$$

Assume $t=1$. Depending on the value of $p$ we have five subcases: $p<-1$, $p=-1,-1<p<1, p=1$ and $p>1$. Respective solutions are given in this order: $\left\{e^{-y} \cos \lambda x, e^{-y} \sin \lambda x, e^{y} \cos \mu x, e^{y} \sin \mu x\right\}$ where $\lambda^{2}=1-p$ and $\mu^{2}=-1-p ;\left\{e^{y}, x e^{y}, e^{-y} \cos \sqrt{2} x, e^{-y} \sin \sqrt{2} x\right\} ;\left\{e^{-y} \cos \lambda x, e^{-y} \sin \lambda x\right.$, $\left.e^{\mu x+y}, e^{-\mu x+y}\right\}$ where $\lambda^{2}=1-p$ and $\mu^{2}=p+1 ;\left\{e^{-y}, x e^{-y}, e^{\sqrt{2} x+y}\right.$, $\left.e^{-\sqrt{2} x+y}\right\}$ and $\left\{e^{\lambda x-y}, e^{-\lambda x-y}, e^{\mu x+y}, e^{-\mu x+y}\right\}$ where $\lambda^{2}=p-1$ and $\mu^{2}=p+1$. Surfaces are listed also in this order

$$
\begin{array}{ll}
22^{\circ} & \tan ^{-1} Z=k \tan ^{-1} \frac{Y}{X}, \quad k>1 \\
23^{\circ} & Z=\tan ^{-1} \frac{Y}{X} \\
24^{\circ} & \tan ^{-1} Z=k \log \frac{Y}{X}, \quad k>0 \\
25^{\circ} & Z=Y e^{X} \\
26^{\circ} & Z=\left(\frac{Y}{X}\right)^{k}, \quad 0<k<1 .
\end{array}
$$

When $t=-1$, the solutions are $\left\{e^{\lambda x} \cos \left(\frac{x}{2 \lambda}+y\right), e^{\lambda x} \sin \left(\frac{x}{2 \lambda}+y\right), e^{-\lambda x}\right.$ $\left.\cos \left(-\frac{x}{2 \lambda}+y\right), e^{-\lambda x} \sin \left(-\frac{x}{2 \lambda}+y\right)\right\}$ where $2 \lambda^{2}=p+\sqrt{1+p^{2}}$. The resulting surface is

$$
27^{\circ} \quad \log \frac{X^{2}+Y^{2}}{1+Z^{2}}=k\left(\tan ^{-1} \frac{Y}{X}-\tan ^{-1} Z\right), \quad k>0
$$


As is easily seen by looking at solutions, all surfaces are homogeneous.

\section{$\S 4$. Remarks}

4.1 Summarizing calculations in previeous sections we have seen that nondegenerate projectively homogeneous surfaces are projectively congruent to one of

$$
0^{0} \quad Z=X^{2}+Y^{2}
$$

and surfaces in the list $1^{\circ} \sim 27^{\circ}$ :

$1^{\circ} \quad Z=Y^{2}+\varepsilon X^{k} \quad k \neq 0,1,2$

$2^{o} \quad Z=Y^{2}+\varepsilon e^{X}$

$3^{\circ} \quad Z=Y^{2}+\varepsilon X \log X$

$4^{o} \quad \tan ^{-1} Z=k \log \left(\frac{X^{2}-\varepsilon Y^{2}}{1+Z^{2}}\right) \quad k \neq 0$

$5^{\circ} \quad e^{Z}=X^{2}+\varepsilon Y^{2}$

$6^{\circ} \quad Z^{k}=X^{2}-Y^{2} \quad k>2$ when $\varepsilon=1$ and $2>k>1$ when $\varepsilon=-1$

$7^{\circ} \quad Z=Y^{2}-\varepsilon \log X$

$8^{\circ} \quad Z^{k}=X^{2}+Y^{2} \quad k>2$ when $\varepsilon=-1$ and $2>k>1$ when $\varepsilon=1$

$9^{\circ} \quad Z+\log \left(X^{2}+Y^{2}\right)=k \tan ^{-1} \frac{Y}{X}, \quad k>0$

$10^{\circ} \quad Z=\frac{1}{\lambda-1} \log X-\frac{1}{\lambda+1} \log Y$

$11^{\circ} \quad \log \frac{X^{2}+Y^{2}}{1+Z^{2}}=k \tan ^{-1} \frac{Y}{X}+\ell \tan ^{-1} Z$

$12^{\circ} \quad \log Z=k \tan ^{-1} \frac{Y}{X}+\frac{\ell}{2} \log \left(X^{2}+Y^{2}\right)$

$13^{\circ} \quad Z=X^{k} Y^{\ell}$

14 $\quad\left(Y-X^{2}\right)^{3}=k\left(Z-\frac{2}{3} X^{3}-X Y\right)^{2}, k \neq 0, \frac{9}{4}$ 
$15^{\circ} \quad Z=X Y+X \log X$

$16^{\circ} \quad Z=X Y+\left(1+X^{2}\right) \tan ^{-1} X$

$17^{\circ} . Z=X Y+\frac{1}{3} X^{3}$

$18^{\circ} \quad Z=X Y+X^{k}, \quad k \neq 0,2$

$19^{\circ} \quad Z=X Y+X^{2} \log X$

$20^{\circ} \quad Z=X Y+e^{X}$

$21^{\circ} \quad \log \frac{X+Y Z}{1+Z^{2}}=k \tan ^{-1} Z, \quad k>0$

$22^{\circ} \quad \tan ^{-1} Z=k \tan ^{-1} \frac{Y}{X}, \quad k>1$

$23^{\circ} \quad Z=\tan ^{-1} \frac{Y}{X}$

$24^{\circ} \quad \tan ^{-1} Z=k \log \frac{Y}{X}, \quad k>0$

$25^{\circ} \quad Z=Y e^{X}$

$26^{\circ} \quad Z=\left(\frac{Y}{X}\right)^{k}, \quad 0<k<1$

$27^{\circ} \quad \log \frac{X^{2}+Y^{2}}{1+Z^{2}}=k\left(\tan ^{-1} \frac{Y}{X}-\tan ^{-1} Z\right), \quad k>0$.

Remark that the above list does not contain degenerate projectively homogeneous surfaces such as projective planes and a surface mentioned in the end of $\S 3$.

4.2 In the above list we find some duplications: The surface $6^{\circ}$ is included in $13^{\circ}$ when $k=\ell$. Similarly $24^{\circ}=12^{\circ}(\ell=0), 26^{\circ}=13^{\circ}(k=-\ell)$, $27^{\circ}=11^{\circ}\left(k^{2}=\ell^{2}\right)$ and $17^{\circ}=18^{\circ}(k=3)$. These are due to the nonuniqueness of the frames we have chosen (each surface could have several but finite number of frames with the required property) and due to that the exact range of the parameters are not determined. For example the surface $Z=X^{k} Y^{\ell}$ is equivalent to $Z=X^{1-k-\ell} Y^{\ell}$ by a transformation $(X, Y, Z) \rightarrow$ $(1 / X, Y / X, Z / X)$. However to understand surfaces geometrically, those 
subcases mentioned above should be excluded from the case written in the right side of equalities.

4.3 In [L], Lie has given, other than quadratic surfaces and the Cayley surface $17^{\circ}, 11$ surfaces with two-dimensional automorphism group over C. They are denoted by I to VII and I' to IV'. Those are identified with surfaces considered over $\mathbb{C}$ in the above list as follows:

$$
\begin{aligned}
& \mathrm{I}=14^{\circ}, \mathrm{II}=1^{\circ}, \mathrm{III}=3^{\circ}, \mathrm{IV}=20^{\circ}, \mathrm{V}=19^{\circ} \\
& \mathrm{VI}=18^{\circ}, \mathrm{VII}=2^{\circ}, \mathrm{I}^{\prime}=7^{\circ}, \mathrm{II}^{\prime}=15^{\circ}, \mathrm{III}^{\prime}=10^{\circ}+5^{\circ}, \mathrm{IV}^{\prime}=13^{\circ} .
\end{aligned}
$$

4.4 In the projective study of surfaces we know several notions such as projective minimality, Demoulin surfaces etc. In regard of these notions we can examine following facts: The Enriques surface $14^{\circ}$ is known to be an affine sphere and, so, projectively minimal. Surfaces in the classes [5] and [6] are Demoulin and projectively minimal. We see surfaces in [3] and [4] are also projectively minimal. Surfaces in [4] are not Demoulin. Surfaces in [2] are projectively minimal only when $|b|=2$, i.e. $k=-2$ or $\frac{2}{3}$ in $1^{o}$. We omit the details.

\section{REFERENCES}

[G] H. W. Guggenheimer, "Differential Geometry," McGraw-Hill, 1963.

[L] S. Lie, Bestimmung aller Flächen, die eine continuirliche Schar von projectiven Transformationen gestatten, Berichte Verh. Ges. Wiss. Leibzig 47 (1895), 209-260.

[S] T. Sasaki, Projective differential geometry and linear homogeneous differential equations, lecture notes at Brown Univ. 1988/89.

[W] E.J. Wilczynski, On a certain class of self-projective surfaces, Trans. Amer. Math. Soc. 14 (1913), 421-443.

(Feb. 1990) 